Prepublication draft.

To appear in IEEE Transactions on Knowledge and Data Engineering

\title{
Multi-Strategy Ensemble Learning: Reducing Error by Combining Ensemble Learning Techniques
}

\author{
Geoffrey I. Webb (author for correspondence) \\ School of Computer Science and Software Engineering \\ Monash University, Clayton, Victoria, 3800, Australia \\ webb@infotech.monash.edu.au \\ Zijian Zheng \\ IDS Software Systems \\ 1065 E. Hillsdale Boulevard, Suite 220, Foster City, CA 94404 \\ zijian@acm.org
}

\begin{abstract}
Ensemble learning strategies, especially Boosting and Bagging decision trees, have demonstrated impressive capacities to improve the prediction accuracy of base learning algorithms. Further gains have been demonstrated by strategies that combine simple ensemble formation approaches. In this paper, we investigate the hypothesis that the improvement in accuracy of multi-strategy approaches to ensemble learning is due to an increase in the diversity of ensemble members that are formed. In addition, guided by this hypothesis, we develop three new multi-strategy ensemble learning techniques. Experimental results in a wide variety of natural domains suggest that these multi-strategy ensemble learning techniques are, on average, more accurate than their component ensemble learning techniques.
\end{abstract}

Index Terms: Boosting, Bagging, Ensemble Learning, Committee Learning, MultiBoosting, Bias, Variance, Ensemble Diversity 


\section{Introduction}

Classification ensemble learning techniques have demonstrated powerful capacities to improve upon the classification accuracy of a base learning algorithm. Common to these approaches is the repeated application of the base learning algorithm to a sample derived from the available training data. Each application of the algorithm results in the generation of a new classifier which is added to the ensemble. To classify a new case, each member of the ensemble classifies the case independently of the others and then the resulting votes are aggregated to derive a single final classification.

It has been observed that an important pre-requisite for classification ensemble learning to reduce test error is that it generate a diversity of ensemble members $[7,8,14,15]$. If all ensemble members agree in all of their classifications then the aggregated classification under any reasonable aggregation scheme will be identical to that of any individual ensemble member. Indeed, for ensembles of numeric predictors for which aggregation of predictions is by weighted numeric mean, it has been proven that increasing diversity between ensemble members without increasing their individual test error necessarily results in a decrease in ensemble test error [13]. This result does not extend directly to classification learning, however, as aggregation of classification predictions is not performed usually by weighted numeric mean. Nonetheless, using majority voting between ensemble members in a two class domain, if diversity in predictions is maximized (as measured by the variance of the predictions) while maintaining a set test error rate for each individual ensemble member, so long as $e<0.5$ and $t \geq \frac{1}{1-2 e}$ rounded up to the next odd integer (where $e$ is the test error for individual ensemble members and $t$ is the ensemble size) the test error rate of the ensemble will be zero. This is because variance will be maximized when $e$ proportion of ensemble members make an error on each test case to be classified. If $e$ is less than 0.5 then this will ensure that the majority vote favors the correct class for every case. The constraint on $t$ is required to ensure that the rounding up of $e$ required by the granularity of ensemble votes still results in a value less than 0.5 for each case to be classified. Turning from this theoretical result, in general, when the test error rate of individual classifiers is less than 0.5, increasing diversity in classifications between classifiers will tend to decrease test error as it will tend to dilute concentrations of errors to less than 0.5 of the votes for any given test case to be classified, hence tending to result in correct classification. 
However, this insight is of less practical value for the generation of classification ensemble learning techniques than might at first be thought. This is because methods for increasing diversity within an ensemble usually come at a cost of also increasing the expected test error of the individual ensemble members. Without knowing the magnitude of the increase in the test error of the individual ensemble members it is not possible to realistically assess the likely outcome of a particular trade-off between diversity and individual error. Assessing the likely increase in individual error is not practical, however, as error estimation on the training data is likely to produce highly optimistic estimates. Nonetheless, the spectacular success of ensemble techniques demonstrates that they manage this trade-off successfully in practice.

With these issues in mind, Webb [20] hypothesized that it would be advantageous to combine ensemble learning techniques that have the capacity to manage effectively this trade-off because doing so will lead to further increases in internal diversity without undue increases in individual error and this can be expected to result in improved classification accuracy. These hypotheses led to the development of MultiBoosting [20], a technique that combines AdaBoost [10] and a variant of Bagging [4] called Wagging [2].

MultiBoosting has been demonstrated to attain most of Boosting's superior bias reduction together with most of Wagging's superior variance reduction. However, which mechanisms are responsible for this outcome remains an open question. This paper investigates the link in multi-strategy ensemble learning between test error reduction and the generation of diversity in ensemble membership. Further, Boosting and Bagging/Wagging are not the only approaches to classification ensemble learning. This paper also explores the effect of increasing the diversity in ensemble membership by integrating the formation of ensembles by stochastic perturbation $[9,1,21]$ with Boosting and Wagging.

\section{Explanations for the effectiveness of ensem- bling}

The spectacular success of ensemble learning has lead to a number of investigations into the underlying mechanisms that support their powerful error reduction capabilities.

Breiman [5] argues that bagging can be viewed as classifying by appli- 
cation of an estimate of the central tendency for the base learner. This may serve to explain why bagging reduces variance. However, it is yet to be explained why such a reduction in variance should not be accompanied by a corresponding increase in error due to bias. Nonetheless, several studies have shown bagging to decrease variance without unduly affecting bias $[7,18,2,20]$.

A contrasting account of the performance of AdaBoost is provided by Friedman, Hastie and Tibshirani [11]. They provide an account of AdaBoost in terms of additive logistic regression. They assert that boosting by reweighting "appears to be a purely 'bias' reduction procedure, intended to increase the flexibility of stable (highly biased) weak learners." Despite this account, a number of empirical studies have demonstrated AdaBoost to reduce both bias and variance [7, 18, 2, 20].

Two sets of studies with artificial data have shown AdaBoost to outperform bagging both in terms of bias and variance reduction [7, 18]. However, experiments with 'natural' data sets seem to indicate that, in general, while AdaBoost is more effective at reducing bias than is bagging, bagging is the more effective at reducing variance $[2,20]$.

Freund and Schapire [10] prove that AdaBoost reduces error on the training data. However, they also note that this need not reduce error outside the training data. They suggest that structural risk minimization [19] might explain off-training set error reduction. However, subsequent empirical evidence has not supported this supposition [18].

Schapire et al. [18] attribute AdaBoost's ability to reduce off-training set error to its boosting the margins of the ensemble's weighted classifications. However, as evidence against this account, Breiman [6] has presented algorithms that are more effective than AdaBoost at increasing margins but less effective at reducing test error.

Breiman [7] ascribes AdaBoost's error reduction to adaptive resampling. This is the construction of an ensemble by repeated sampling from a training set where the probability of a training case being included in a subsequent sample is increased if it has been misclassified by ensemble members learned from previous samples. Some support for this argument is provided by the success of an alternative adaptive resampling algorithm, arc-x4. However, while AdaBoost has been demonstrated to be equally effective at reducing error using either reweighting or resampling, arc-x4 has been shown to be much less effective using reweighting than using resampling [2]. This could be taken to indicate that AdaBoost does more than just adaptive resampling. 
As can be seen, while investigation into the success of ensemble learning techniques has been extensive, no single account has received undisputed widespread support. In this context this paper investigates the role of diversity between ensemble members in the effectiveness of ensemble learning.

\section{Ensemble learning techniques}

This section describes the ensemble learning techniques utilized in this work. All of the techniques take a base learning algorithm and a set of training data and then repeatedly apply the algorithm or a variant thereof to a sample from the data, producing a set of classifiers. These classifiers then vote to reach an ensemble classification.

Bagging applies the base algorithm to bootstrap samples from the training data. A bootstrap sample from $n$ cases is formed by randomly selecting $n$ cases with replacement. Wagging is similar to Bagging except that all training cases are retained in each training set, but each case is stochastically assigned a weight. In the current research we follow Webb [20] in assigning weights from the continuous Poisson distribution (more commonly known as the exponential distribution). This is motivated by the observation that Bagging can be considered to be Wagging with allocation of weights from the discrete Poisson distribution, and hence the use of the continuous Poisson distribution provides a natural extension of Bagging to utilization of fractional weights. Individual random instance weights (approximately) conforming to the continuous Poisson distribution are calculated by the following formula:

$$
\operatorname{Poisson}()=-\log \left(\frac{\operatorname{Rand}(1 \ldots 999)}{1000}\right)
$$

where $\operatorname{Rand}(x \ldots y)$ returns a random integer value between $x$ and $y$ inclusive.

The resulting algorithm is presented as Algorithm 1. In general, Wagging is slightly less effective than Bagging at test error reduction, perhaps because the inclusion of every case in every training set tends to lead to lower variation between the resulting ensemble members [20]. Nonetheless, we utilize Wagging rather than Bagging in the current research as it interacts better with other ensemble learning algorithms, possibly because it includes all training cases, allowing the other algorithm access to all cases on every run. 


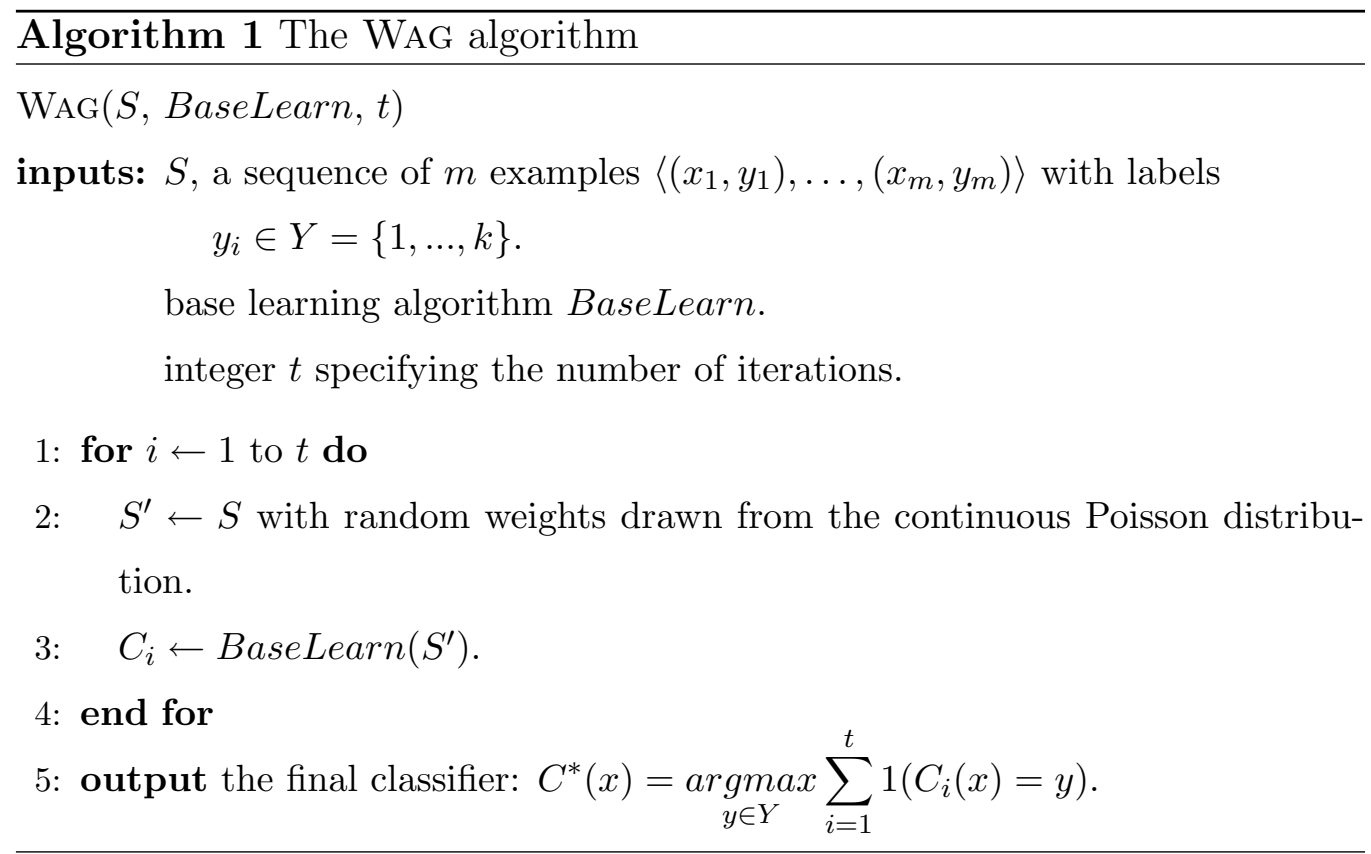

Boosting is another approach to ensemble learning. The first ensemble member is formed by applying the base learning algorithm to the entire training set. Subsequent ensemble members are formed by applying the base algorithm to the training set but with cases reweighted to place higher weight on cases that are misclassified by existing ensemble members. The votes of ensemble members are weighted by a function that lowers the vote of a classifier that has lower accuracy on the weighted training set from which it was learned. We utilize a minor variant on Bauer and Kohavi's [2] variant of Freund and Schapire's [10] AdaBoost algorithm. This is presented as Algorithm 2. Bauer and Kohavi's [2] variant

- Uses a one step weight update process that is less subject to numeric underflow than the original two step process (step 17).

- Prevents numeric underflow (step 18).

- Continues producing more ensemble members beyond the point where $\epsilon \geq 0.5$ (step 5). This measure is claimed to improve prediction accuracy $[5,2]$.

We further modify this approach by utilizing the continuous Poisson distribution for reweighting cases at steps 6 and 12 and continuing to produce more 
ensemble members beyond a point where training error falls to zero (step 10). These two measures are included for the sake of consistency between the various learning algorithms, all use the continuous Poisson distribution for random reweighting and all always produce an ensemble of size $t$. Note that this version of the algorithm may fail to terminate, entering an infinite loop through steps 3 to 8 . This did not occur in our experiments.

Stochastic Attribute Selection Committee Learning differs from the above techniques in that instead of perturbing the training set, it performs stochastic perturbations to the base learning algorithm on successive applications of the learner to a training set. A number of variations on this general approach have been explored $[9,1]$. While our specific technique (described in more detail by Zheng and Webb [21]) differs in minor details from these previous approaches, we have no reason to believe that, in general, its performance would differ substantially from the alternatives. Our implementation, that uses C4.5 [16] Release 8 as the base learning algorithm, operates as follows. When learning a decision tree, C4.5 applies an information measure to each potential test selecting the test with the highest value of a criterion based on gain ratio [17]. C4.5SAS modifies this behaviour by introducing a stochastic element to the selection process, allowing tests with lower values on the selection criterion to occasionally be selected. This is achieved by selecting a subset of the available attributes, with each available attribute having probability of 0.33 of inclusion. If there is an acceptable test on the attributes included in the subset then the one that rates highest on the selection criterion is selected. If there is no such test among the selected attributes, the best test among all attributes is selected unless there is no acceptable test in which case a leaf is formed.

Our implementation of Stochastic Attribute Selection Committees, SAsc, repeatedly applies C4.5SAS to the training data to create an ensemble of classifiers. This process is presented as Algorithm 3.

\section{Multi-Strategy Ensemble Learning Algorithms}

We combine multiple approaches to ensemble learning motivated by the hypothesis that doing so will increase diversity between ensemble members, albeit at a cost of a small increase in individual error. Webb [20] hypothesized that this process would trade-off diversity against individual error so 
Algorithm 2 The AdaBoost algorithm

$\operatorname{AdaBoost}(S$, BaseLearn, $t)$

inputs: $S$, a sequence of $m$ examples $\left\langle\left(x_{1}, y_{1}\right), \ldots,\left(x_{m}, y_{m}\right)\right\rangle$ with labels

$$
y_{i} \in Y=\{1, \ldots, k\} \text {. }
$$

base learning algorithm BaseLearn.

integer $t$ specifying the number of iterations.

1: $S^{\prime} \leftarrow S$ with all instance weights set to 1 .

2: for $i=1$ to $t$ do

3: $\quad C_{i} \leftarrow$ BaseLearn $\left(S^{\prime}\right)$.

4: $\quad \epsilon_{i} \leftarrow \frac{\sum_{x_{j} \in S^{\prime}: C_{i}\left(x_{j}\right) \neq y_{j}} \text { weight }\left(x_{j}\right)}{m}$ \{the weighted error on the training set\}

5: if $\epsilon_{i}>0.5$ then

6: $\quad$ reset $S^{\prime}$ to random weights from the continuous Poisson distribution.

7: $\quad$ standardize $S^{\prime}$ to sum to $m$.

8: $\quad$ goto step 3 .

9: $\quad$ end if

10: if $\epsilon_{i}=0$ then

11: $\quad$ set $\beta_{i}$ to $10^{-10}$

12: $\quad$ reset $S^{\prime}$ to random weights from the continuous Poisson distribution.

13: $\quad$ standardize $S^{\prime}$ to sum to $m$.

14: else

15: $\quad \beta_{i} \leftarrow \frac{\epsilon_{i}}{\left(1-\epsilon_{i}\right)}$.

16: $\quad$ for all $x_{j} \in S^{\prime}$ do

17: $\quad$ divide weight $\left(x_{j}\right)$ by $2 \epsilon_{i}$ if $C_{i}\left(x_{j}\right) \neq y_{j}$ and $2\left(1-\epsilon_{i}\right)$ otherwise.

18: $\quad$ if weight $\left(x_{j}\right)<10^{-8}$, set weight $\left(x_{j}\right)$ to $10^{-8}$.

19: $\quad$ end for

20: end if

21: end for

22: output the final classifier: $C^{*}(x)=\underset{y \in Y}{\operatorname{argmax}} \sum_{i: C_{i}(x)=y} \log \frac{1}{\beta_{i}}$. 
Algorithm 3 The SASC learning algorithm

$\operatorname{SAsC}(A t t, D, P, t)$

inputs: Att: a set of attributes,

$D$ : a training set represented using Att and classes,

$P$ : the probability with which an attribute should be included in

the set of attributes available at a node,

$t$ : the number of trials.

1: for $i \leftarrow 1$ to $t$ do

2: $\quad C_{i} \leftarrow \mathbf{C 4 . 5 S A S}(A t t, D, P)$

3: end for

4: output the final classifier: $C^{*}(x)=\underset{y \in Y}{\operatorname{argmax}} \sum_{i=1}^{t} 1\left(C_{i}(x)=y\right)$.

as to decrease the resulting ensemble's test error. In the current work we seek to evaluate this hypothesis and explore the role of ensemble member diversity in ensemble learning.

MultiBoosting has established that the combination of Boosting and Wagging can reduce test error. However, this does not answer the questions of whether this reduction can be attributed to an increase in the diversity of ensemble members or whether combinations of other forms of ensemble learners may also be productive. We address the latter question by exploring the space of combinations of Boosting, Wagging, and Stochastic Attribute Selection Committees. For our experimental work we utilize the well known C4.5 Release 8 [17] as the base learning algorithm.

To combine SASC with another method we replace C4.5 with C4.5SAS as the base learning algorithm within the other method. To combine Wagging with Boosting we follow Webb's [20] MultiBoosting approach, Wagging sub-ensembles formed by Boosting. The MB algorithm is presented in Algorithm 4. Note that for consistency with the other approaches to combining base learning algorithms, we have modified MB to utilize stochastic weights for the first sub-ensemble (step 1) rather than initializing all weights to 1 as done by Webb [20]. Note also that this version of the algorithm, as is the case with our version of AdaBoost, may fail to terminate, entering an infinite 
loop through steps 9 to 15. This did not occur in our experiments.

Together with the two base learning algorithms, C4.5 and C4.5SAS, combining the algorithms in all possible ways results in nine distinct algorithms, presented in Figure 1.

\section{Experiments}

We use the following notation:

$Y$ is the set of classes.

$\mathcal{T}$ is a training set of example description-classification pairs.

$K$ is a classifier, a function from objects to classes.

$C_{i}$ is the $i^{\text {th }}$ classifier in ensemble $C$, a function from objects to classes.

$W_{i}$ is the weight given to the vote of $C_{i}$.

$t$ is the number of classifiers in ensemble $C$.

$x_{i}$ is the description of the $i^{\text {th }}$ case to be classified.

$y_{i}$ is the correct classification for the $i^{\text {th }}$ case to be classified.

$m$ is the number of cases to be classified.

$\mathcal{L}$ is a learner, a function from training sets to classifiers.

We wish to evaluate the hypothesis that combining multiple distinct ensemble learning algorithms will tend to increase diversity in the predictions of ensemble members without unduly increasing the test error of the individual predictions of the ensemble members, resulting in a reduction in ensemble test error.

To evaluate this hypothesis we need an operational measure of diversity in the predictions of ensemble members. We utilize the weighted statistical variance between the weighted predictions of the members of a classifier ensemble for this purpose:

$$
\text { diversity }=\frac{\sum_{i=1}^{m}\left(1-\sum_{y \in Y}\left(\frac{\sum_{j=1}^{t} W_{j} 1\left(C_{j}\left(x_{i}\right)=y\right)}{\sum_{j=1}^{t} W_{j}}\right)^{2}\right)}{m}
$$




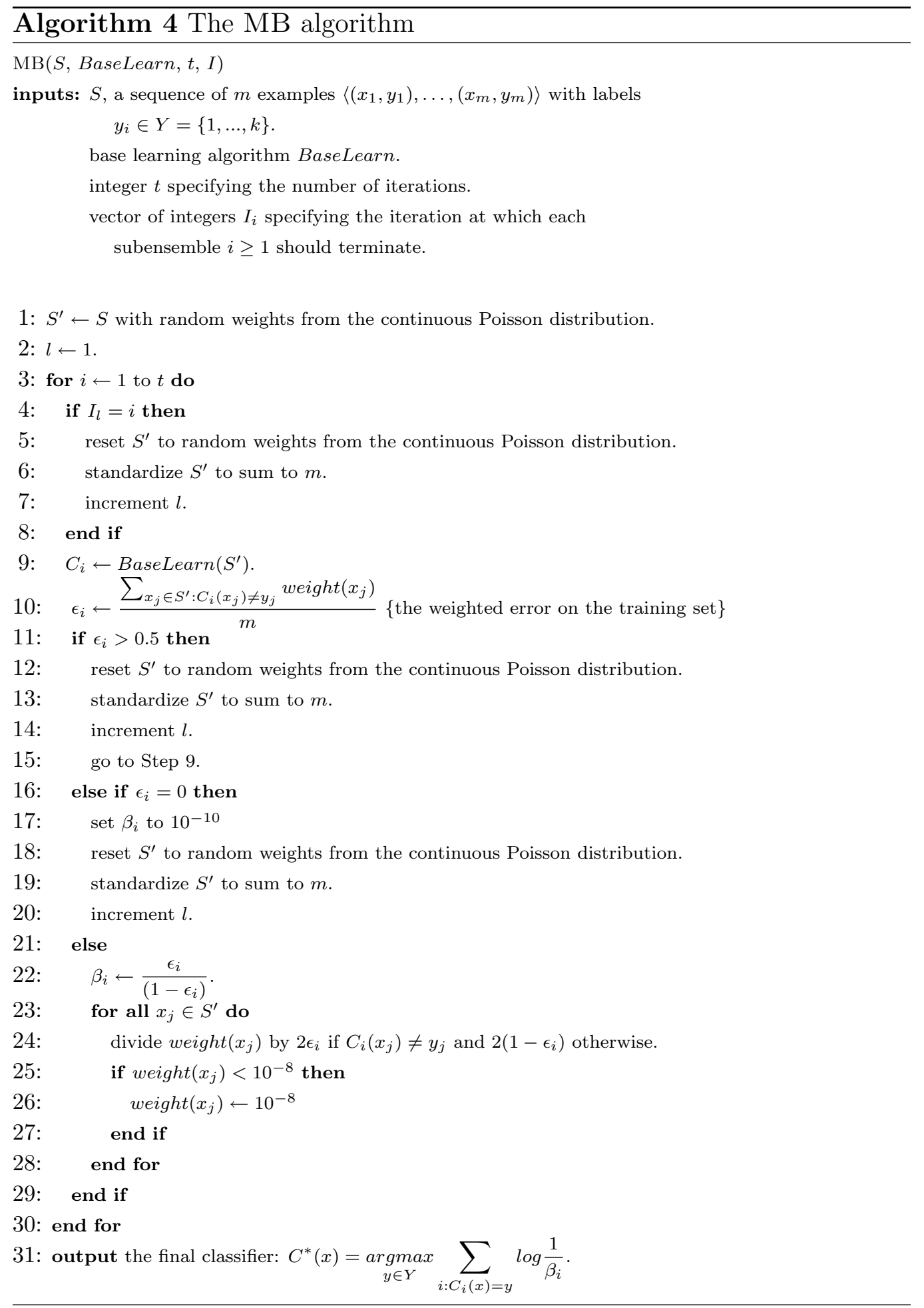


We also require an operational measure of the test error of the individual predictions of ensemble members. We utilize a weighted mean of the test error of the predictions of an ensemble's constituent classifiers for this purpose:

$$
\text { individual error }=\frac{\sum_{i=1}^{m} \sum_{j=1}^{t} W_{j} 1\left(C_{j}\left(x_{i}\right)=y_{i}\right)}{m \sum_{j=1}^{t} W_{j}}
$$

We wish to examine the relationship between these two measures and error, which we define as

$$
\text { error }=\frac{\sum_{i=1}^{m} 1\left(K\left(x_{i}\right) \neq y_{i}\right)}{m}
$$

We further decompose error into bias and variance using Kohavi and Wolpert's [12] definitions thereof:

$$
\begin{gathered}
\text { bias }_{x}^{2}=\frac{1}{2} \sum_{y \in Y}\left[P_{Y, X}(Y=y \mid X=x)-P_{\mathcal{T}}(\mathcal{L}(\mathcal{T})(x)=y)\right]^{2} \\
\text { variance }_{x}=\frac{1}{2}\left(1-\sum_{y \in Y} P_{\mathcal{T}}(\mathcal{L}(\mathcal{T})(x)=y)^{2}\right) \\
\sigma_{x}=\frac{1}{2}\left(1-\sum_{y \in Y} P_{Y, X}(Y=y \mid X=x)^{2}\right)
\end{gathered}
$$

The third term $\sigma$ relates to irreducible error. We follow Kohavi and Wolpert's [12] practice of aggregating this value with bias due to the difficulty of estimating it from observations of classification performance.

We estimate all of the above terms using Webb's [20] procedure of performing ten cycles of three-fold cross validation. This process ensures that each case is used in twenty training sets and ten test sets.

Armed with these measures and procedures we systematically explored the space of combinations of the three base ensemble learning algorithms by forming the following systems that realize the hierarchy illustrated in Figure 1:

C4.5: C4.5 Release 8, the base system.

C4.5SAS: C4.5 modified to perform stochastic attribute selection as described in Section 3. 
WAG: Wagged ensembles of 100 decision trees using C4.5 as the base algorithm.

Boost: AdaBoost ensembles of 100 decision trees using $\mathrm{C} 4.5$ as the base algorithm.

SASC: Stochastic attribute selection committees of 100 decision trees each formed by C4.5SAS.

MB: MultiBoosted (Wagged subensembles formed by boosting) ensembles of $100(10 \times$ subensembles of size 10) decision trees using $\mathrm{C} 4.5$ as the base algorithm.

BoostSAsc: Boosted ensembles of 100 decision trees using C4.5SAS as the base algorithm.

WaGSASC: Wagged ensembles of 100 decision trees using C4.5SAS as the base algorithm.

MBSASC: MultiBoosted ensembles of $100(10 \times$ subensembles of size 10) decision trees using $\mathrm{C} 4.5 \mathrm{SAS}$ as the base algorithm.

These various algorithms were applied to the 41 data sets from the UCI repository [3] described in Appendix A. Ensembles of size 100 were used as a compromise between greater compute times required by larger ensembles and the ever decreasing average-case marginal improvement in error that can be expected from larger ensemble sizes.

Unfortunately, space constraints prevent the presentation of results at the individual dataset level. Figures 2 to 6 and Tables 1 to 5 provide high level summaries of these results. The summary tables have the following format, where row indicates the mean value on a data set for the algorithm with which a row is labeled, while col indicates the mean value for the algorithm with which the column is labeled. Rows labeled $\dot{\mathrm{r}}$ present the geometric mean of the value ratio col/row. Rows labeled $s$ present the win/draw/loss record, where the first value is the number of data sets for which col < row, the second is the number for which col = row and the last is the number for which col $>$ row. Rows labeled $p$ present the result of a twotailed sign test on the win-loss record. This is the two-tailed probability of obtaining the observed record of wins to losses, or more extreme, if wins and losses were equi-probable random events. Note, these values have not 
been corrected to control experiment-wise type-1 error, but are in most cases so low that standard statistical corrections would not affect significance test outcomes. The figures depict the hierarchy of Figure 1, with mean value across all data sets listed against each algorithm. The lines climbing up the hierarchy are labeled with an indication of the relative win/draw/loss record. An improvement in the win/draw/loss record is labeled with a ' + ' and a decline by a '-.' Where the difference is statistically significant at the 0.05 level the label is large, otherwise it is small.

\subsection{Error, Bias and Variance}

Figure 2 shows that the mean error invariably drops as we climb the hierarchy of ensemble technique combinations. Table 1 shows that all ensemble techniques have significantly better win/draw/loss records than C4.5. Moving from a single strategy to a combination of two strategies, in every case the error ratio and win/draw/loss record favors the multi-strategy approach over each of its constituent strategies. The win/draw/loss record significantly favors the multi-strategy approach over the constituent strategy in every case except for comparing Boost against BoostSasc. Moving from combinations of two strategies to the combination of all three strategies, the error ratio and win/draw/loss record in each case favors MBSASC, but the advantage on the win/draw/loss record is only significant against BoOsTSASC. While the failure to obtain significant advantages at the top of the hierarchy leaves room for interpretation about whether MBSASC holds a general advantage over each of its two-strategy constituents, it at the very least appears clear that it does not hold a significant general disadvantage.

Turning to bias, all of the ensemble techniques have lower bias, favorable bias ratios and favorable win/draw/loss records with respect to C4.5. However, the win/draw/loss records only indicate significant benefits for the ensemble techniques that incorporate boosting as a component technique. Climbing the hierarchy, MB shows a marginally worse aggregate mean bias and bias ratio relative to Boost and the win/draw/loss record approaches significance at the 0.05 level. In contrast, BoostSASC shows marginal improvements in mean bias and bias ratio, but the very small advantage in win/draw/loss record is definitely not significant. Relative to SASC, BoOsTSASC demonstrates clear wins on all metrics, but WAGSASC demonstrates only a minor win on mean bias, no win on bias ratio, and an extremely slim, 


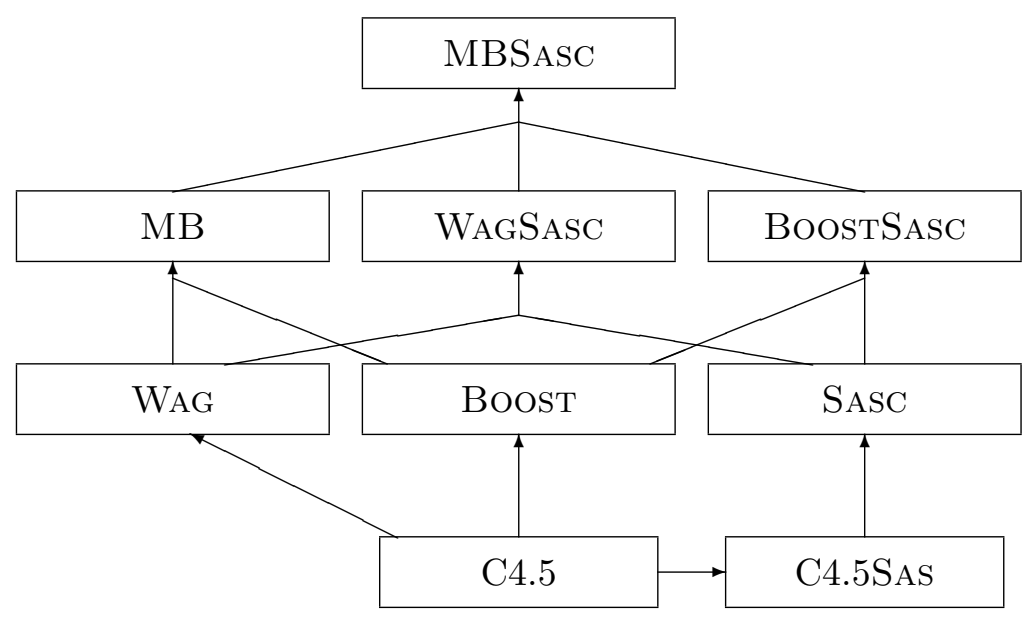

Figure 1: Hierarchy of ensemble learning technique combinations

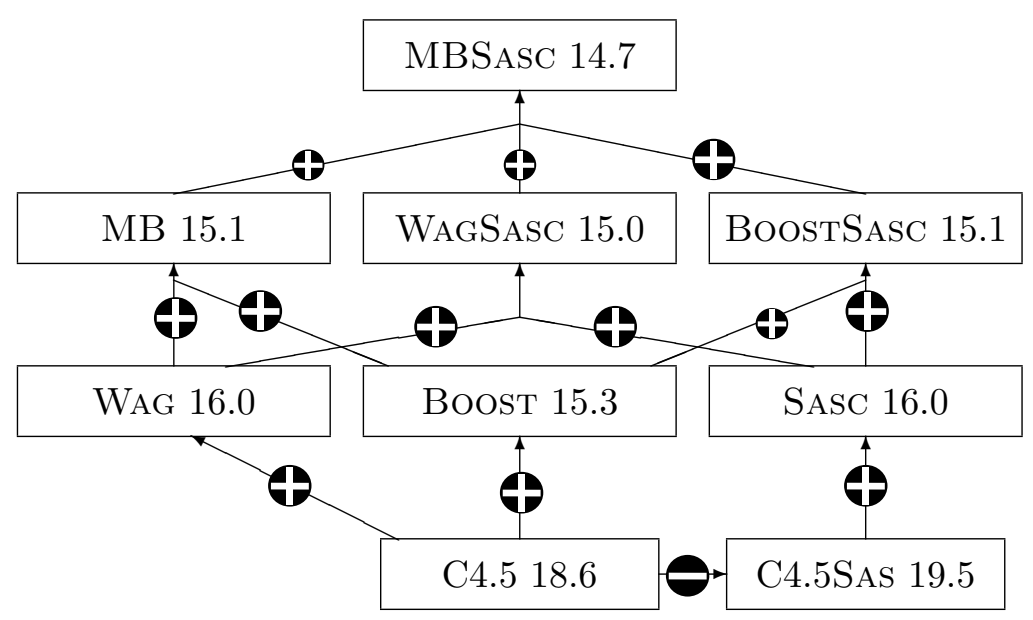

Figure 2: Hierarchy of error outcomes 
Table 1: Error rate comparison results

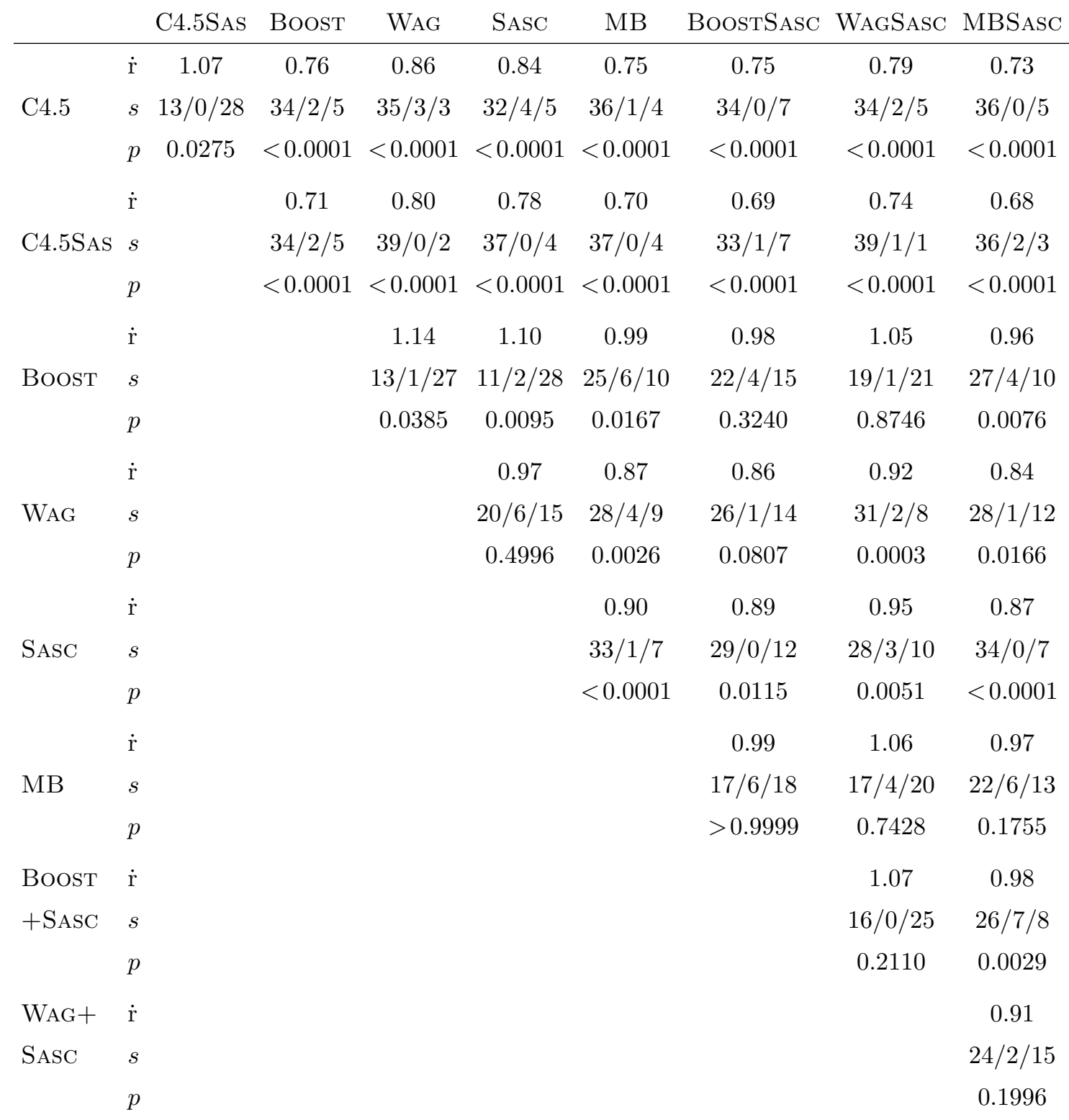


Table 2: Bias comparison results

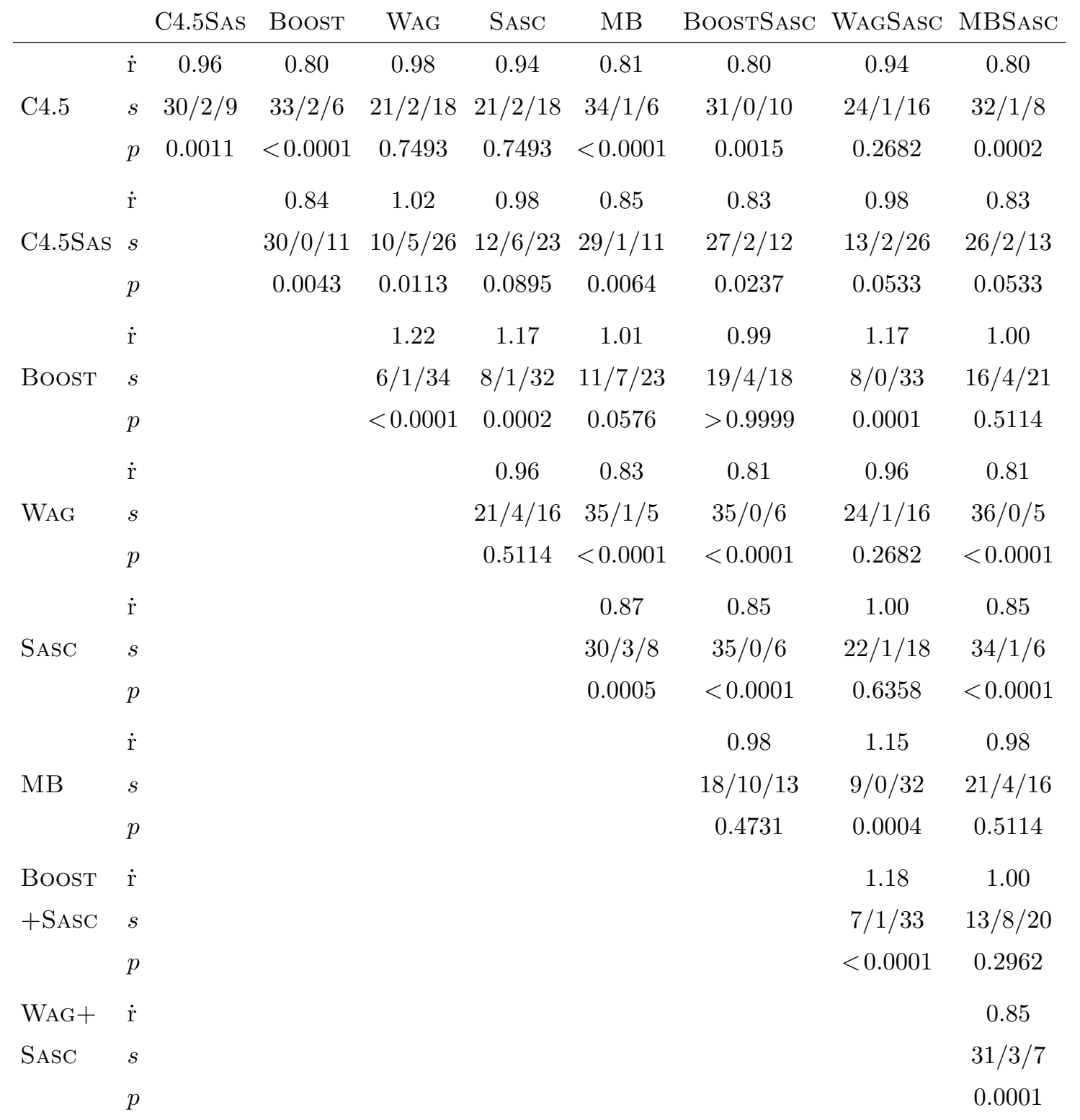


far from significant win on win/draw/loss record. With respect to WAG, MB demonstrates clear wins on all metrics while WAGSASC demonstrates marginal wins and the win on win/draw/loss record is not significant. Proceeding to the top of the hierarchy, MBSASC demonstrates a clear win over WAGSASC on all metrics, but has at most marginal, non-significant, wins against MB and actually has a worse win/drawn/loss record, albeit not significantly, than BoostSasc. In summary, combining Boosting with Wagging or Stochastic Attribute Selection appears to have a beneficial effect with respect to bias, but the combination of Wagging with Stochastic Attribute Selection appears to have little effect in this respect.

Turning our attention next to variance, a very different pattern appears. Again, all of the ensemble techniques outperform C4.5 on all metrics. Adding either WAG or SASC to another technique, including each other, always produces a substantial benefit on all metrics, including a significant improvement in win/draw/loss record relative to the other technique on its own. In contrast, however, adding Boost to another technique always results in a decrease in performance on all metrics (excepting small, non-significant, improvements in win/draw/loss record for MB against WAG and BoostSASC against SASC), sometimes a substantial decrease and in one case (MBSASC against WAGSASC) a significant worsening of the win/draw/loss record.

In summary, these results suggest that Boost is primarily a bias reduction technique. Although it performs significant variance reduction, it is not as effective at this as WAG or SASC. Combining Boost with WAG or SASC produces significant benefits in bias reduction over each of WAG and SASC in isolation, without a serious decline vis a vis Boost. In contrast, WAG and SASC are primarily variance reduction techniques. Combining either with another technique results in variance reduction vis a vis the other technique on its own without a serious increase in variance in comparison to WAG or SASC in isolation. Putting all of these factors together, combining techniques is generally beneficial with respect to error reduction because there is always a benefit either in terms of bias or variance reduction against each constituent technique, which is usually gained without a substantial or significant loss with respect to the other constituent of error. 


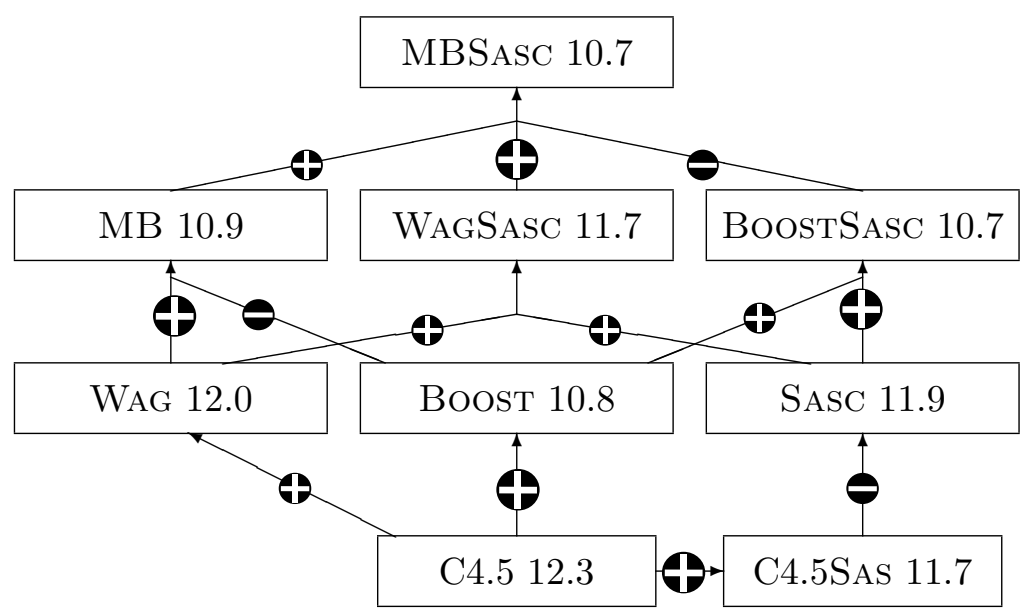

Figure 3: Hierarchy of bias outcomes

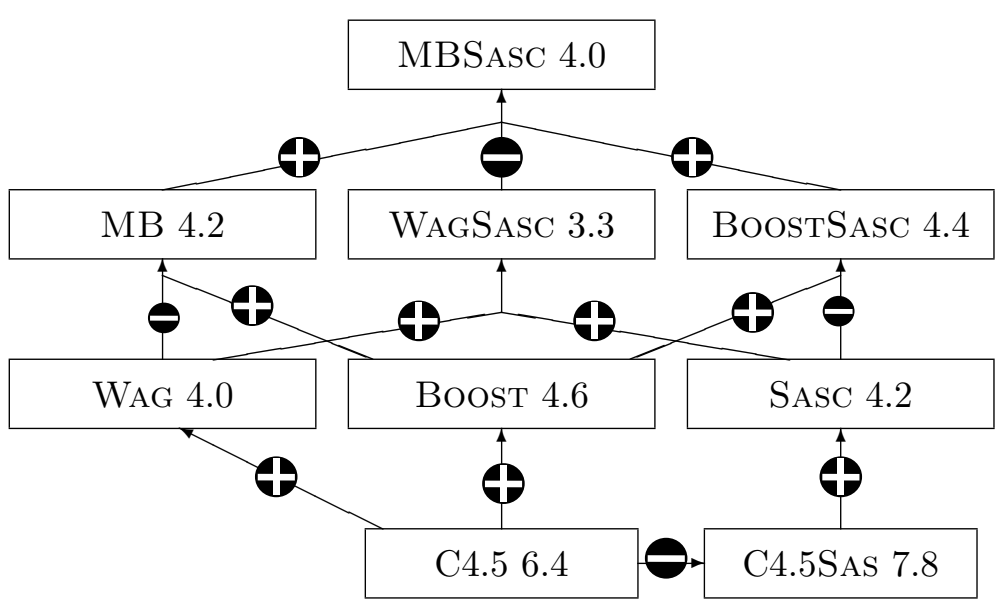

Figure 4: Hierarchy of variance outcomes 
Table 3: Variance comparison results

\begin{tabular}{|c|c|c|c|c|c|c|c|c|c|}
\hline & & C4.5SAS & Boost & WAG & SASC & MB & BoostSasc & WAGSASC & MBSASC \\
\hline & $\dot{\mathrm{r}}$ & 1.25 & 0.75 & 0.65 & 0.60 & 0.69 & 0.72 & 0.51 & 0.65 \\
\hline \multirow[t]{3}{*}{ C4.5 } & $s$ & $6 / 2 / 33$ & $34 / 2 / 5$ & $38 / 2 / 1$ & $37 / 3 / 1$ & $34 / 2 / 5$ & $33 / 3 / 5$ & $37 / 3 / 1$ & $34 / 3 / 4$ \\
\hline & $p$ & $<0.0001$ & $<0.0001$ & $<0.0001$ & $<0.0001$ & $<0.0001$ & $<0.0001$ & $<0.0001$ & $<0.0001$ \\
\hline & $\dot{\mathrm{r}}$ & & 0.60 & 0.53 & 0.48 & 0.56 & 0.58 & 0.41 & 0.52 \\
\hline \multirow[t]{3}{*}{ C4.5SAS } & $s$ & & $36 / 0 / 5$ & $40 / 0 / 1$ & $39 / 0 / 2$ & $37 / 0 / 4$ & $36 / 0 / 5$ & $40 / 0 / 1$ & $38 / 0 / 3$ \\
\hline & $p$ & & $<0.0001$ & $<0.0001$ & $<0.0001$ & $<0.0001$ & $<0.0001$ & $<0.0001$ & $<0.0001$ \\
\hline & $\dot{\mathrm{r}}$ & & & 0.87 & 0.80 & 0.93 & 0.96 & 0.68 & 0.87 \\
\hline \multirow[t]{3}{*}{ Boost } & $s$ & & & $20 / 5 / 16$ & $18 / 5 / 18$ & $31 / 9 / 1$ & $21 / 11 / 9$ & $31 / 3 / 7$ & $32 / 6 / 3$ \\
\hline & $p$ & & & 0.6177 & $>0.9999$ & $<0.0001$ & 0.0428 & 0.0001 & $<0.0001$ \\
\hline & $\dot{\mathrm{r}}$ & & & & 0.92 & 1.06 & 1.10 & 0.78 & 1.00 \\
\hline \multirow[t]{3}{*}{ WAG } & $s$ & & & & $21 / 5 / 15$ & $19 / 6 / 16$ & $18 / 5 / 18$ & $34 / 4 / 3$ & $21 / 5 / 15$ \\
\hline & $p$ & & & & 0.4050 & 0.7359 & $>0.9999$ & $<0.0001$ & 0.4050 \\
\hline & $\dot{\mathrm{r}}$ & & & & & 1.16 & 1.20 & 0.85 & 1.09 \\
\hline \multirow[t]{3}{*}{ SASC } & $s$ & & & & & $21 / 8 / 12$ & $21 / 7 / 13$ & $29 / 9 / 3$ & $26 / 4 / 11$ \\
\hline & $p$ & & & & & 0.1628 & 0.2295 & $<0.0001$ & 0.0201 \\
\hline & $\dot{\mathrm{r}}$ & & & & & & 1.03 & 0.74 & 0.94 \\
\hline \multirow[t]{2}{*}{ MB } & $s$ & & & & & & $11 / 10 / 20$ & $30 / 3 / 8$ & $24 / 7 / 10$ \\
\hline & $p$ & & & & & & 0.1496 & 0.0005 & 0.0243 \\
\hline Boost & $\dot{\mathrm{r}}$ & & & & & & & 0.71 & 0.91 \\
\hline \multirow[t]{2}{*}{$+\mathrm{SASC}$} & $s$ & & & & & & & $31 / 4 / 6$ & $32 / 7 / 2$ \\
\hline & $p$ & & & & & & & $<0.0001$ & $<0.0001$ \\
\hline WAG+ & $\dot{\mathrm{r}}$ & & & & & & & & 1.27 \\
\hline \multirow[t]{2}{*}{ SASC } & $s$ & & & & & & & & $12 / 4 / 25$ \\
\hline & $p$ & & & & & & & & 0.0470 \\
\hline
\end{tabular}




\subsection{Diversity and individual error}

Webb's [20] original motivation for the development of MultiBoosting and the subsequent multi-strategy ensemble learning techniques was that combining ensemble learning strategies would increase diversity without unduly affecting the error of individual members of the ensemble. Tables 4 and 5 show that this is indeed the case when one considers every step from a constituent ensemble learning technique to a multi-strategy technique except for Boost to MB and BoostSasc to MBSasc. The steps from Wag to MB, Boost to BoostSasc, Sasc to BoostSasc, Wag to WagSasc, Sasc to WagSasc, MB to MBSAsc, and WagSasc to MBSASC all result in increases in diversity accompanied by small but significant increases in individual error but decreases in ensemble error. However, adding WAG to BoosT or MBSASC has the opposite effect on diversity and individual error. In both cases both diversity and individual error significantly decrease. However, in both cases this nonetheless results in a decrease in ensemble error. We have the unexpected outcome that the original motivation for MultiBoosting appears to apply to other combinations of standard classifier ensemble learning techniques, but not to the MultiBoost combination of WAG and Boost!

Having observed this phenomenon, it is straightforward to explain. Compared with WAG and SAsc, Boost has very high diversity. It is credible that the Boosting process will tend to drive diversity up at ever increasing rates as ensemble size increases. This is due to the manner in which Boosting attempts to focus the learner on areas of the instance space that previous ensemble members fail to handle adequately. By definition, this means that it is attempting to make latter ensemble members systematically differ in their classifications from prior members. However, this process will also drive up the individual error of each successive ensemble member when applied to the domain as-a-whole, because each successive member concentrates primarily on ever decreasing areas of the total instance space. Successive ensemble members have ever increasing individual error in order to gain the ever increasing diversity, a trade-off that in practice results in ever diminishing benefit in terms of reduction of overall ensemble error. Credibility is lent to this argument when one compares the diversity and individual error of AdaBoost ensembles of size ten and 100. To this end, the AdaBoost experiments were rerun, on the same data set cross-validation partitions, but with an ensemble size of ten. With respect to diversity, in contrast to the mean across all data sets of 0.340 obtained for boosted ensembles of size 100, boosted ensembles 


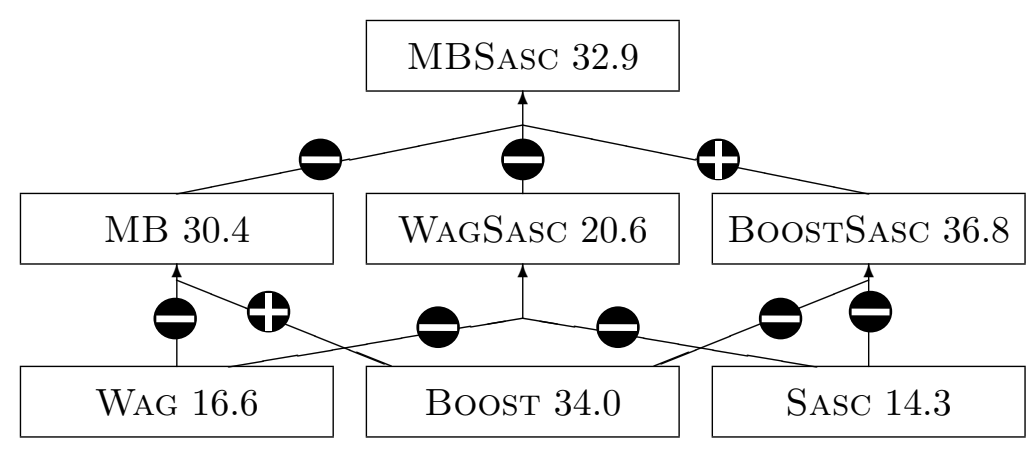

Figure 5: Hierarchy of diversity outcomes

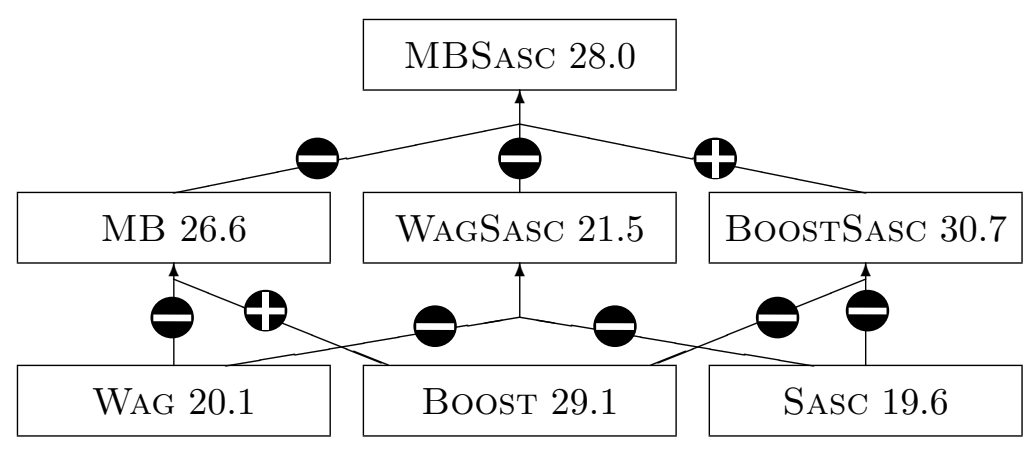

Figure 6: Hierarchy of individual error outcomes 
Table 4: Diversity comparison results

\begin{tabular}{|c|c|c|c|c|c|c|c|}
\hline & & WAG & SASC & $\mathrm{MB}$ & BoostSAsC & WAGSASC & MBSASC \\
\hline \multirow{3}{*}{ Boost } & $\dot{\mathrm{r}}$ & 0.37 & 0.29 & 0.85 & 1.09 & 0.47 & 0.93 \\
\hline & $s$ & $41 / 0 / 0$ & $41 / 0 / 0$ & $41 / 0 / 0$ & $2 / 0 / 39$ & $41 / 0 / 0$ & $28 / 1 / 12$ \\
\hline & $p$ & $<0.0001$ & $<0.0001$ & $<0.0001$ & $<0.0001$ & $<0.0001$ & 0.0166 \\
\hline \multirow{3}{*}{ WAG } & $\dot{\mathrm{r}}$ & & 0.78 & 2.30 & 2.96 & 1.29 & 2.51 \\
\hline & $s$ & & $26 / 1 / 14$ & $0 / 0 / 41$ & $0 / 0 / 41$ & $1 / 1 / 39$ & $0 / 0 / 41$ \\
\hline & $p$ & & 0.0807 & $<0.0001$ & $<0.0001$ & $<0.0001$ & $<0.0001$ \\
\hline \multirow{3}{*}{ SASC } & $\dot{\mathrm{r}}$ & & & 2.95 & 3.79 & 1.65 & 3.22 \\
\hline & $s$ & & & $0 / 0 / 41$ & $0 / 0 / 41$ & $0 / 0 / 41$ & $0 / 0 / 41$ \\
\hline & $p$ & & & $<0.0001$ & $<0.0001$ & $<0.0001$ & $<0.0001$ \\
\hline \multirow{3}{*}{ MB } & $\dot{\mathrm{r}}$ & & & & 1.29 & 0.56 & 1.09 \\
\hline & $s$ & & & & $0 / 0 / 41$ & $41 / 0 / 0$ & $1 / 0 / 40$ \\
\hline & $p$ & & & & $<0.0001$ & $<0.0001$ & $<0.0001$ \\
\hline Boost & $\dot{\mathrm{r}}$ & & & & & 0.43 & 0.85 \\
\hline \multirow[t]{2}{*}{$+\mathrm{SASC}$} & $s$ & & & & & $41 / 0 / 0$ & $41 / 0 / 0$ \\
\hline & $p$ & & & & & $<0.0001$ & $<0.0001$ \\
\hline WAG + & $\dot{\mathrm{r}}$ & & & & & & 1.95 \\
\hline \multirow[t]{2}{*}{ SASC } & $s$ & & & & & & $0 / 0 / 41$ \\
\hline & $p$ & & & & & & $<0.0001$ \\
\hline
\end{tabular}


Table 5: Individual error rate comparison results

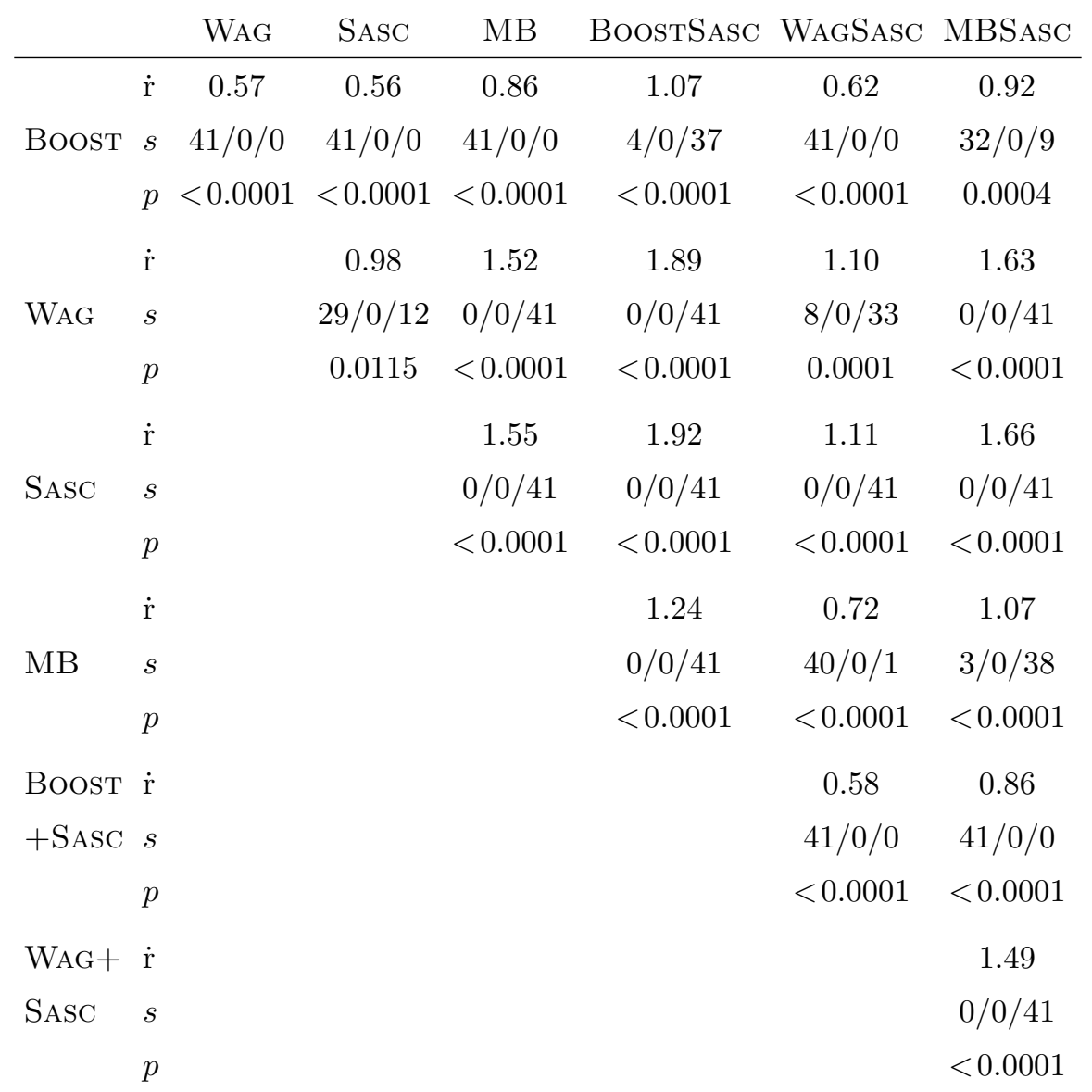


of size ten had a mean of 0.276 . With respect to individual error, the mean dropped from 0.291 to 0.260 for ensembles of size ten. With respect to both measures, the mean on every data set was lower for ensemble size ten than for size 100 .

MultiBoosting creates boosted sub-ensembles of size ten. Compared with the mean diversity and individual error obtained for boosted ensembles of this size, MultiBoosting does lead to increases. However, it is clear that MultiBoosting compared with Boosting ensembles of size 100 leads to decreases in diversity and individual error. By creating boosted sub-ensembles of size ten, MultiBoosting delivers lower internal error than Boosting. However, this improvement in internal error comes at a cost of a slight decrease in diversity. Contrary to our expectations, rather than increasing diversity vis a vis Boosting, MultiBoosting decreases diversity, but, in practice does so in a manner that forms a better diversity against individual error trade-off than that formed by Boosting alone.

Other than these two cases where Wagging is combined with Boosting, the results are, however, consistent with our expectations that combining ensemble techniques would result in increased diversity and individual error resulting in trade-offs that reduce overall ensemble error.

\section{Conclusions}

This paper has examined techniques for combining simple ensemble learning approaches with the aim of exploring the relationship between ensemble member diversity and ensemble error. The results strongly support the proposition that combining effective ensemble learning strategies is conducive to reducing test error. A specific hypothesis about this effect was examinedthat combining ensemble learning strategies would increase diversity at the cost of a small increase in individual test error resulting in a trade-off that reduced overall ensemble test error. While this hypothesis was consistent with the results obtained when stochastic attribute selection was combined with another ensemble learning strategy, it was not consistent with the results for the MultiBoosting approach to combining Boosting and Wagging, where, compared with the Boosting-based strategy (AdaBoost alone, or AdaBoost combined with SASC) the combination appears to have the effect of reducing individual test error at the cost of a small reduction in diversity, a different 
trade-off which nonetheless results in reduced ensemble test error.

The success of these ensemble learning techniques mandates further investigation. We have examined only a single base learner and only one ensemble size. Our expectation is that the results will generalize to other base learners and ensemble sizes but this belief warrants evaluation. This paper has highlighted the trade-off between diversity and individual test error in ensemble learning strategies. Research into how this trade-off should be managed and how to identify when a particular trade-off is likely to be productive are likely prove fruitful areas for future investigation.

The computational overheads of combining ensemble learning strategies are negligible. The same number of ensemble members are learned, and hence the same numbers of calls to the base learner are required. Indeed, the strategy of wagging sub-committees formed by boosting can support greater computational efficiency by allowing parallelism of a form not readily possible with boosting alone. However, despite negligible computational cost, our experiments have shown that appreciable and reasonably consistent reductions in test error can be obtained. There appears to be no reason not to combine ensemble learning strategies in a learning scenario for which ensemble learning is appropriate.

\section{Acknowledgments}

The Breast Cancer (S), and Lymphography data sets were provided by the Ljubljana Oncology Institute, Slovenia. Thanks to the UCI Repository's maintainers and donors, for providing access to the data sets used herein.

\section{A Data sets}

Forty one natural domains from the UCI machine learning repository are used. Table 6 summarizes the characteristics of these domains, including dataset size, the number of classes, the number of numeric attributes, and the number of discrete attributes. This test suite covers a wide variety of different domains with respect to dataset size, the number of classes, the number of attributes, and types of attributes. 
Table 6: Description of learning tasks

\begin{tabular}{|c|c|c|c|c|c|c|c|c|c|}
\hline \multirow[t]{2}{*}{ Domain } & \multirow[t]{2}{*}{ Size } & \multirow{2}{*}{$\begin{array}{c}\text { No. of } \\
\text { Classes }\end{array}$} & \multicolumn{2}{|c|}{ No. of Att. } & \multirow[t]{2}{*}{ Domain } & \multirow[t]{2}{*}{ Size } & \multirow{2}{*}{$\begin{array}{c}\text { No. of } \\
\text { Classes }\end{array}$} & \multicolumn{2}{|c|}{ No. of Att. } \\
\hline & & & Numeric & Discr & & & & Numeric & Discr \\
\hline Adult & 48842 & 4 & 6 & 7 & Labor & 57 & 2 & 8 & 8 \\
\hline Annealing & 898 & 6 & 6 & 32 & LED 24 & 200 & 10 & 0 & 24 \\
\hline Audiology & 226 & 24 & 0 & 69 & Letter & 20000 & 26 & 16 & 0 \\
\hline Automobile & 205 & 7 & 15 & 10 & Liver disorders & 345 & 2 & 6 & 0 \\
\hline Balance scale & 625 & 3 & 4 & 0 & Lymphography & 148 & 4 & 0 & 18 \\
\hline Breast (S) & 286 & 2 & 0 & 9 & NetTalk letter & 5438 & 163 & 0 & 7 \\
\hline Breast (W) & 699 & 2 & 9 & 0 & NetTalk stress & 5438 & 5 & 0 & 7 \\
\hline Chess (KR-KP) & 3169 & 2 & 0 & 36 & NetTalk phoneme & 5438 & 52 & 0 & 7 \\
\hline Credit (A) & 690 & 2 & 6 & 9 & New thyroid & 215 & 3 & 5 & 0 \\
\hline Credit (G) & 1000 & 2 & 7 & 13 & Pima diabetes & 768 & 2 & 8 & 0 \\
\hline Discordant & 3772 & 2 & 7 & 22 & Postoperative & 90 & 3 & 1 & 7 \\
\hline Echocardiogram & 131 & 2 & 6 & 1 & Promoters & 106 & 2 & 0 & 57 \\
\hline Glass & 214 & 6 & 9 & 0 & Segment & 2310 & 7 & 19 & 0 \\
\hline Heart & 270 & 2 & 7 & 6 & Sick & 3772 & 2 & 7 & 22 \\
\hline Heart (C) & 303 & 2 & 13 & 0 & Sonar & 208 & 2 & 60 & 0 \\
\hline Heart (H) & 294 & 2 & 13 & 0 & Soybean large & 683 & 19 & 0 & 35 \\
\hline Hepatitis & 155 & 2 & 6 & 13 & Splice junction & 3177 & 3 & 0 & 60 \\
\hline Horse colic & 368 & 2 & 7 & 15 & Vehicle & 846 & 4 & 18 & 0 \\
\hline House votes 84 & 435 & 2 & 0 & 16 & Waveform-21 & 300 & 3 & 21 & 0 \\
\hline Нуро & 3772 & 5 & 7 & 22 & Wine & 178 & 3 & 13 & 0 \\
\hline Iris & 150 & 3 & 4 & 0 & & & & & \\
\hline
\end{tabular}




\section{References}

[1] K. Ali. Learning Probabilistic Relational Concept Descriptions. PhD thesis, Dept. Information and Computer Science, Univ. of California, Irvine, 1996.

[2] E. Bauer and R. Kohavi. An empirical comparison of voting classification algorithms: Bagging, boosting, and variants. Machine Learning, 36:105139, 1999.

[3] C. Blake and C. J. Merz. UCI repository of machine learning databases. [Machine-readable data repository]. Univ. of California, Dept. Information and Computer Science, Irvine, CA., 2001.

[4] L. Breiman. Bagging predictors. Machine Learning, 24:123-140, 1996.

[5] L. Breiman. Bias, variance, and arcing classifiers. tech. report 460, Statistics Dept., Univ. of California, Berkeley, CA, 1996.

[6] L. Breiman. Arcing the edge. tech. report 486, Statistics Dept., Univ. of California, Berkeley, CA, 1997.

[7] L. Breiman. Arcing classifiers. The Annals of Statistics, 26(3):801-849, 1998.

[8] T. G. Dietterich. An experimental comparison of three methods for constructing ensembles of decision trees: Bagging, boosting, and randomization. Machine Learning, 40(2):139-158, 2000.

[9] T. G. Dietterich and E. B. Kong. Machine learning bias, statistical bias, and statistical variance of decision tree algorithms. Tech. report, Dept. Computer Science, Oregon State University, 1995.

[10] Y. Freund and R. E. Schapire. A decision-theoretic generalization of on-line learning and an application to boosting. Journal of Computer and System Sciences, 55(1):119-139, 1997.

[11] J. H. Friedman, T. Hastie, and R. Tibshirani. Additive logistic regression: A statistical view of boosting. Annals of Statistics, 28(2), 2000. 
[12] R. Kohavi and D. Wolpert. Bias plus variance decomposition for zeroone loss functions. Proc. 13th Int'll. Conf. Machine Learning, pp. 275283, 1996. Morgan Kaufmann.

[13] A. Krogh and J. Vedelsby. Neural network ensembles, cross validation, and active learning, G. Tesauro, D. Touretzky, and T. Leen, Eds., Advances in Neural Information Processing Systems, vol. 7, pp. 231-238. MIT Press, 1995.

[14] S. Lee and J. F. Elder. Bundling Heterogeneous Classifiers with Advisor Perceptrons, tech report 97-1, Elder Research, Charlottesville, VA, 1997.

[15] D. Margineantu and T. G. Dietterich. Pruning Adaptive Boosting, Proc. 14th Int'l. Conf. Machine Learning (ICML-97), pp.211-218, 1997. Morgan Kaufmann.

[16] J. R. Quinlan. C4.5: Programs for Machine Learning. Morgan Kaufmann, San Mateo, CA, 1993.

[17] J. R. Quinlan. Improved use of continuous attributes in C4.5. Journal of Artificial Intelligence Research, 4:77-90, 1996.

[18] R. E. Schapire, Y. Freund, P. Bartlett, and W. S. Lee. Boosting the margin: A new explanation for the effectiveness of voting methods. The Annals of Statistics, 26(5):1651-1686, October 1998.

[19] V. N. Vapnik. Estimation of Dependencies Based on Empirical Data. Springer-Verlag, 1982.

[20] G. I. Webb. MultiBoosting: A technique for combining boosting and wagging. Machine Learning, 40(2):159-196, 2000.

[21] Z. Zheng and G. I. Webb. Stochastic attribute selection committees. Proc. 11th Australian Joint Conf. Artificial Intelligence, pp. 321-332, 1998. Springer. 